\title{
El valor del agua que producen los bosques y cafetales en la subcuenca del río Acelhuate
}

\author{
José Ricardo Calles-Hernández' \\ Recibido: 09/05/2014 - Aceptado: 07/06/2014
}

\begin{abstract}
Resumen
El valor del agua que producen los ecosistemas forestados puede estimarse a partir de una valoración económica-ecológica, en el entendido de que el recurso hídrico es un insumo de la producción para los distintos sectores económicos en un determinado territorio. Una valoración de este tipo, también debe conducir a reconocer los costos de oportunidad en los que incurren los dueños de las tierras en donde se alojan estos ecosistemas; y además, los costos que supondría restaurar las áreas de recarga hídrica para posibilitar que el servicio hídrico sea continuo y de calidad. Este artículo presenta una metodología que emplea estas consideraciones, para calcular el valor del agua que proveen los ecosistemas boscosos y cafetales de la subcuenca del río Acelhuate, lo cual debiera traducirse en precios de referencia, pagaderos por los distintos usuarios, como reconocimiento al servicio ambiental hídrico que proveen estos ecosistemas.

\section{Palabras clave}

Ecosistema, sectores económicos, recarga hídrica, valor del agua.
\end{abstract}

\section{Abstract}

The value of the water produced by the forest ecosystems can be estimated from an economic and ecological valuation, with the understanding that the water resource is a production input for the different economic sectors in a given territory. An assessment of this kind should also lead to recognizing the opportunity costs incurred by landowners where these ecosystems are housed, plus the estimated costs for the restoration of water recharge areas to enable a continuous water service of quality. This paper presents a methodology that uses these considerations to calculate the value of the water provided by forest ecosystems and coffee plantations in the Acelhuate River watershed, which should result in reference prices payable for different users, in recognition of the water environmental service provided by these ecosystems.

\section{Keywords}

Ecosystems, economic sectors, water supply, water value. 


\section{Introducción}

La subcuenca del río Acelhuate se encuentra circunscrita dentro de la cuenca del río Lempa (región hidrográfica " $A$ "), posee una superficie de $717.35 \mathrm{~km}^{2}$, y presenta los siguientes límites geográficos: al norte con el margen derecho del río Lempa, al sur con el complejo volcánico de la cordillera del Bálsamo, al oeste está determinada por el complejo volcánico del volcán San Salvador, correspondiente a la cadena volcánica central, y al es te con la caldera volcánica de Ilopango y las subcuencas Copinolapa y Quezalapa (figura 1).

\section{Figura 1. Subcuenca del río Acelhuate}

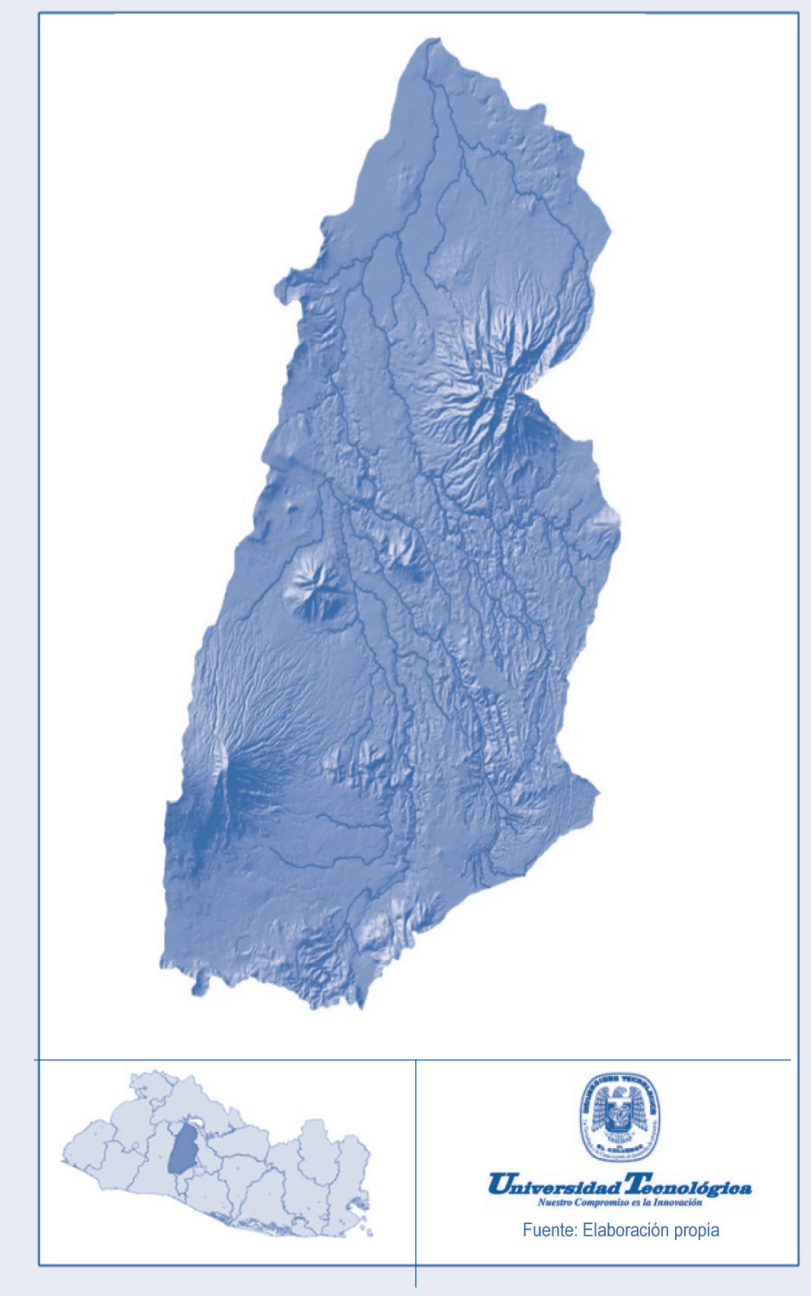

Esta subcuenca es uno de los territorios hidrológicos de El Salvador con mayores presiones socioeconómicas, lo cual ha desencadenado procesos complejos de degradación ambiental a todo nivel, incluyendo la calidad y cantidad del recurso hídrico. A pesar de ello, existen en la subcuenca importantes áreas de recarga ocupadas por ecosistemas de bosques y cafetales, los cuales posibilitan el abastecimiento de agua en el área metropolitana de San Salvador.

Hasta ahora, el servicio ecosistémico de provisión hídrica que generan estas áreas forestadas no posee un valor monetario que pueda ser reconocido por parte de los usuarios del agua, mediante un sistema de "cobro y pago" por el beneficio que reciben al disponer de este líquido para las distintas actividades económicas. Por tanto, para alcanzar una gestión integral del agua y sostenibilidad en su provisión, se requiere que los sectores económicos del territorio internalicen en sus costos un valor monetario del servicio ambiental hídrico, cuyo monto puede ser estimado a través de una metodología de valoración económica, en vista de que el mercado no asigna un precio a dicho servicio, ni a las alteraciones que este sufre por efecto, entre otras causas, de la intervención humana.

La valoración económica ecológica que se presenta en este artículo considera los siguientes aspectos: (i) la función de captación hídrica; (ii) los costos de restauración de ecosistemas; y (iii) el agua como insumo de la producción.

\section{Captación hídrica de ecosistemas}

La captación hídrica hace referencia a la capacidad que tienen los bosques y cafetales de influir sobre las tasas de infiltración de los suelos, lo cual, a su vez, reduce las tasas de escorrentía superficial y mejora el flujo de agua subterráneo, incidiendo en la temporalidad de los caudales de los ríos y las pérdidas de nutrientes y sedimentos (Jobbágy, 2009). Los ecosistemas forestados localizados en la subcuenca del río Acelhuate se encuentran influyendo sobre la cantidad y calidad del agua que circula en este territorio hidrológico.

En otras palabras, el bosque y los cafetales de la subcuenca permiten que se potencie la infiltración del agua, y hacen que esta se encuentre disponible para que la sociedad pueda aprovecharla en las distintas actividades socioeconómicas. Como muestra la tabla 1, en un terreno con cobertura boscosa se verifican mejores tasas de infiltración de un volumen de agua determinado (en función del tiempo); en promedio, el bosque es capaz de infiltrar agua a una tasa del $68,92 \%$ en un plazo de 1 hora, en comparación con una cobertura bajo pasto, que lo hace a $24,75 \%$, y un suelo sin cobertura vegetal, que infiltra el 6,33\%. 
Tabla 1.

Infiltración del agua en terrenos con diferentes coberturas

\begin{tabular}{|c|c|c|c|c|c|c|c|}
\hline \multirow{2}{*}{$\begin{array}{c}\text { Tiempo } \\
\text { en minutos }\end{array}$} & \multicolumn{2}{|c|}{ Cobertura boscosa } & \multicolumn{2}{|c|}{ Cobertura bajo pasto } & \multicolumn{2}{|c|}{ Suelo sin cobertura vegetal } & \multirow{2}{*}{$\begin{array}{l}\text { Total } \\
\left(\mathrm{cm}^{3}\right)\end{array}$} \\
\hline & $\left(\mathrm{cm}^{3}\right)$ & $\%$ & $\left(\mathrm{~cm}^{3}\right)$ & $\%$ & $\left(\mathrm{~cm}^{3}\right)$ & $\%$ & \\
\hline 5 & 60.00 & 69,52 & 21.00 & 24,33 & 5.30 & 6,14 & 86.30 \\
\hline 10 & 119.00 & 67,70 & 45.80 & 26,05 & 11.00 & 6,26 & 175.80 \\
\hline 30 & 360.00 & 68,90 & 127.00 & 24,31 & 35.50 & 6,79 & 522.50 \\
\hline 60 & 715.00 & 69,55 & 250.00 & 24,32 & 63.00 & 6,13 & 1.028 .0 \\
\hline Promedio & & 68,92 & & 24,75 & & 6,33 & 0 \\
\hline
\end{tabular}

Fuente: Heuveldop et al., 1986

Según el Centro Agronómico Tropical de Investigación y Enseñanza (Catie, 1997), el valor de infiltración neta en un bosque tropical oscila entre 9.000 a $14.000 \mathrm{~m}^{3} / \mathrm{ha} / \mathrm{año}$; mientras que CCT-Cinterpeds (1995) demuestra que los bosques pueden amortiguar "picos de agua", disminuir la velocidad del agua y mejorar su calidad (tabla 2).

Entre tanto, la Fundación Salvadoreña para la investigación del Café (Procafe, 2009) considera que el cafetal constituye la mayor reserva boscosa productiva y generadora de servicios ambientales en El Salvador. Según esta institución, los cafetales contribuyen a la retención de sedimentos y de nutrientes que protegen el suelo de la erosión, evitando el empobrecimiento de estos, y el azolvamiento de represas; además, regulan las precipitaciones, evitando que las escorrentías excesivas provoquen deslaves e inundaciones en los valles y zonas costeras.

Tabla 2

Escenario de escorrentía media anual de algunas zonas de vida ( $\mathrm{m}^{3} / \mathrm{ha} / \mathrm{año}$ )

\begin{tabular}{|r|r|r|r|r|r|r|}
\hline \multirow{2}{*}{ Zona de vida } & \multicolumn{3}{|c|}{ Cobertura bosque } & \multicolumn{3}{c|}{ Cobertura pasto } \\
\cline { 2 - 7 } & \multicolumn{1}{|c|}{$\begin{array}{c}\text { Total de } \\
\text { agua por } \\
\text { escorrentía }\end{array}$} & $\begin{array}{c}\text { Calidad } \\
\text { positiva }\end{array}$ & $\begin{array}{c}\text { Calidad } \\
\text { negativa }\end{array}$ & $\begin{array}{c}\text { Total de } \\
\text { agua por } \\
\text { escorrentía }\end{array}$ & $\begin{array}{c}\text { Calidad } \\
\text { positiva }\end{array}$ & $\begin{array}{c}\text { Calidad } \\
\text { negativa }\end{array}$ \\
\hline Tropical húmedo (T-w) & 36.740 .00 & 30.610 .00 & 6.130 .00 & 40.060 .00 & 15.010 .00 & 25.050 .00 \\
\hline Premontano húmedo (P-w) & 18.610 .00 & 16.280 .00 & 2.330 .00 & 21.460 .00 & 7.150 .00 & 14.310 .00 \\
\hline Premontano lluvioso (LM-r) & 42.490 .00 & 31.870 .00 & 10.620 .00 & 44.360 .00 & 11.090 .00 & 33.270 .00 \\
\hline Premontano húmedo (LM-w) & 16.870 .00 & 15.330 .00 & 1.540 .00 & 18.900 .00 & 6.880 .00 & 12.020 .00 \\
\hline Montano húmedo (M-w) & 9.120 .00 & 8.070 .00 & 1.050 .00 & 10.340 .00 & 3.580 .00 & 6.760 .00 \\
\hline Montano lluvioso (M-r) & 20.550 .00 & 15.420 .00 & 5.130 .00 & 20.660 .00 & 5.160 .00 & 15.500 .00 \\
\hline Total & 144.380 .00 & 117.580 .00 & 26.800 .00 & 155.780 .00 & 48.870 .00 & 106.910 .00 \\
\hline Porcentaje & 100,00 & 81,44 & 18,56 & 100,00 & 31,37 & 68,63 \\
\hline
\end{tabular}

Fuente: CCT-CINTERPEDS, 1995.

Dicha fundación también reconoce que los ecosistemas de cafetal contribuyen a la regulación del ciclo hidrológico, favoreciendo la cantidad y distribución de las precipitaciones, alimentado las fuentes de agua superficiales como ríos, lagos y represas hidroeléctricas; y en el caso de los mantos acuíferos, enriqueciéndolos cada 60 minutos con 500 metros cúbicos de agua, aspecto que es clave para el abastecimiento de agua para el consumo humano; así como para el desarrollo de la agricultura y de la industria en general.

Por todos estos beneficios resulta fundamental reconocer el servicio ecosistémico de la productividad hídrica del bosque y cafetal; y más aún, cobrar y pagar un precio por él. Este 
precio deberá representar la importancia que la sociedad reconoce en este servicio ecosistémico, respecto a otros, tales como mitigación del calentamiento global, retención de sedimentos y nutrientes, regulación del régimen de lluvias, reducción del riesgo a inundaciones, recarga hídrica, conservación de biodiversidad y belleza escénica (Martínez, 2008).

El reconocimiento monetario de la sociedad debe concretarse mediante un pago a los propietarios de tierra, cuyo valor deberá ser igual o mayor a la utilidad que estos dejan de obtener por mantener la ocupación del suelo por los ecosistemas forestados. En esta línea de ideas, el enfoque de la valoración económica está referido al "costo de oportunidad" de los dueños de bosques y cafetales en la subcuenca; es decir, que el valor monetario de la captación hídrica que realizan estos ecosistemas debe ser equivalente (o incluso superior) a los ingresos anuales que podrían percibir los propietarios de la tierra por otros usos de suelo, distintos al bosque y el cafetal; es decir, la agricultura, ganadería u ocupaciones urbanísticas.

Por lo tanto, la metodología de valoración toma en cuenta los siguientes aspectos: (i) el volumen anual de agua captada y fijada por los ecosistemas en las zonas de recarga de la subcuenca; (ii) el cálculo del costo de oportunidad del uso de la tierra en esas zonas; y (iii) la ponderación de la importancia del bosque y el cafetal en términos de su productividad hídrica, al compararla con los otros servicios ambientales. La siguiente fórmula permite estimar el valor de captación de los ecosistemas bosque y cafetal:

$$
V C=\frac{\propto \beta A b}{O_{c}} \quad \begin{array}{ll}
\text { ecuación } \\
\text { (1) }
\end{array}
$$

Donde:

VC: Valor de captación hídrica de ecosistemas (US $\left.\$ / m^{3}\right)$.

$\propto$ : Importancia de los ecosistemas en la subcuenca en función de la cantidad y calidad de agua $(0 \leq \propto \leq 1)$.

$\beta$ : Costo de oportunidad de cualquier actividad económica que compite con los ecosistemas por el uso del suelo en la subcuenca (US\$/ha/año).

Ab: Área bajo cobertura de bosque y café en la subcuenca (ha).

$\mathrm{O}_{\mathrm{c}}$ : Volumen de agua captada por bosques de la subcuenca ( $m^{3} /$ ha/año).

\section{Costos de restauración de ecosistemas}

Los costos de restauración de los ecosistemas que se localizan en las zonas de recarga hídrica en la subcuenca del río Acelhuate constituyen una parte del valor monetario del bienestar que perciben los usuarios del agua por el aprovechamiento del servicio ambiental hídrico. Estos costos equivalen a los $\operatorname{costos}^{1}$ de planes, programas y proyectos de restauración de ecosistemas, principalmente en cabeceras de cuencas; y se incurrirá en ellos, hasta alcanzar un nivel de restauración que simule las condiciones de conservación previas a los procesos de degradación.

Debido a que la restauración de ecosistemas no solo potencia el servicio ambiental hídrico, es necesario ponderar el valor monetario en función únicamente de la protección del agua; para ello, se necesita calcular las hectáreas que se deben restaurar y sus respectivos costos por unidad de superficie. Se emplea la siguiente fórmula, para ello:

$$
V R=\frac{\delta C}{O_{c}} \quad \begin{array}{ll}
\text { ecuación } \\
\text { (2) }
\end{array}
$$

Donde:

VR: Valor de restauración de ecosistemas (US\$ $\left./ \mathrm{m}^{3}\right)$ $C$ : Costos de la actividad destinada a la restauración de la subcuenca (US\$/ha/año)

$\delta$ : Fracción del costo destinado a la restauración de ecosistemas en función del recurso hídrico de la subcuenca (\%)

$\mathrm{O}_{c}$ : Volumen de agua captada por bosques de la subcuenca ( $\left.m^{3} / \mathrm{ha} / \mathrm{año}\right)$

\section{Valor del agua como insumo de la producción}

La valoración económica-ecológica incluye, finalmente, el valor del agua como insumo de la producción, el cual, debido a los diferentes usos que hacen los sectores socioeconómicos, deberá determinarse a través de diversas técnicas, las cuales se detallan así:

\section{$\checkmark$ Sectores doméstico, industrial y comercial}

La técnica utilizada en este caso se denomina "excedente del consumidor", la cual hace referencia al nivel de bienestar (utilidad) que logra la sociedad (consumidores) cuando

Incluyen salarios, cargas sociales de personal destinados a esa actividad, gastos en combustibles, transportes, infraestructura y otros costos de operación e incentivos utilizados para la protección ambiental 
los niveles de oferta de un recurso (el agua) cambian, principalmente por efecto del crecimiento poblacional o el mismo desarrollo económico (Calles, 2003), por lo que se requiere estimar la "elasticidad precio de la demanda", e indagar sobre la disponibilidad a pagar (DAP) de los usuarios del agua en los sectores en análisis. Para alcanzar este objetivo, se emplea la función de demanda Cobb-Douglas, cuya expresión es la que se muestra a continuación:

$$
Q=k P^{\varepsilon} \quad \text { ecuación }
$$

Donde:

\section{(3)}

Q: Volumen de agua ( $\mathrm{m}^{3} / \mathrm{mes}$ )

$P$ : Tarifa financiera actual por el servicio de abastecimiento del agua (US\$/m³)

$k$ : Factor de proporcionalidad

$\varepsilon$ : Elasticidad precio de la demanda

Cuando se conoce un par de puntos $\left(\mathrm{Q}_{1}, \mathrm{P}_{1}\right)$ y la elasticidad $\varepsilon_{\mathrm{r}}$ se determina $\mathrm{k}_{1}$ en el período $1\left(\mathrm{D}_{1}\right)$, así:

$$
k_{1}=Q_{1} P_{1}^{-\varepsilon} \quad \text { ecuación }
$$

Según Barrantes y Vega (2002), de la misma forma puede obtenerse una curva de demanda para un segundo período $\left(D_{2}\right)$, suponiendo una tasa de crecimiento $r$, ya sea por efecto del crecimiento poblacional, o sea por un aumento en la renta per cápita. de manera que se cumpla con $k_{t}=k_{1}(1+r)^{t-1}$, donde $t$ representa cualquier otro período futuro.

Al obtener por lo menos dos curvas de demanda $\left(D_{1}\right.$ y $D_{2}$ ), en un escenario en el que la oferta de agua tiende a aumentar (con una inminente subida en el precio del agua por el aumento en el costo de abastecimiento, del primero al segundo período), puede estimarse el nivel de bienestar social (excedente del consumidor) en los sectores doméstico, industrial y comercial, a partir de la siguiente fórmula:

$$
V A=\frac{P_{1}\left(Q_{2}^{\frac{1}{\varepsilon}+1}-Q_{1}^{\frac{1}{\varepsilon}+1}\right)}{Q_{1}^{\frac{1}{\varepsilon}}\left(\frac{1}{\varepsilon}+1\right)}-\mathrm{P}_{2}\left(Q_{2}-Q_{1} \quad\right. \text { (5) }
$$

Donde, $P_{2}\left(Q_{2}-Q_{1}\right)$ representa el costo social del abastecimiento adicional de agua.

\section{Sector agrícola}

La técnica que se debe utilizar en este sector se conoce como "cambio de productividad", cuyo enfoque se basa en el reconocimiento de que el rendimiento de los cultivos incrementa como efecto del riego. Si a ese cambio en producción se le asocia con su precio de mercado, se obtendrá una buena estimación del valor del agua que es utilizada en la agricultura (Barrantes y Vega, 2002). Por lo tanto, para aplicar esta técnica, es necesario disponer de algún registro de incremento de la productividad de los principales cultivos en la subcuenca del río Acelhuate. De ahí que, si el rendimiento de un cultivo $\mathrm{k}$ registra alzas en su producción cuando está bajo riego, es decir:

$$
P_{k}^{a g}=\left(p_{k}-c_{k}\right) \times q_{k} \quad \text { ecuación }
$$

Además: $\left(Q_{\text {riego }}^{k}-Q_{\text {secano }}^{k}\right) / V_{i}$

Donde:

$P_{k}^{a g}$ : Costo del agua en la agricultura para el cultivo k (US\$/ $\left.\mathrm{m}^{3}\right)$

$p_{k}$ : Precio del producto $\mathrm{k}(\mathrm{US} \$ / \mathrm{Kg}$ )

$c_{k}$ : Costo de producción bajo riego (US\$/Kg)

$q_{k}$ : Cambio en producción del cultivo $\mathrm{k}$ bajo riego $\left(\mathrm{Kg} / \mathrm{m}^{3}\right)$

$Q_{\text {riego }}^{k}:$ Cantidad de producción del cultivo k bajo riego $\left(\mathrm{Kg} / \mathrm{m}^{3}\right)$

$Q_{\text {secano }}^{k}:$ Cantidad de producción del cultivo k bajo riego

$(\mathrm{Kg} / \mathrm{Ha})$

$Q_{\text {riego }}^{k}$ : Cantidad de producción del cultivo k sin riego (Kg/Ha) $V_{i}$ : Volumen de agua usado en riego del cultivo $\left(\mathrm{m}^{3} / \mathrm{Ha}\right)$

\section{Valor promedio para el agua}

Con la intención de reflejar un único valor del agua como insumo de la producción, se opta por estimar un valor "promedio ponderado", al cual puede asociársele datos de demanda hídrica y precios por unidad de volumen, utilizando la siguiente expresión matemática:

$$
V P_{a}=\frac{\sum_{i=1}^{n} P_{i} Q_{i}^{d}}{\sum_{i=1}^{n} Q_{i}^{d}}
$$

ecuación

(7) 
Donde:

$\mathrm{VP}_{\mathrm{a}}$ : Valor promedio del agua como insumo de la producción (US\$ $\left./ \mathrm{m}^{3}\right)$

$\mathrm{P}_{\mathrm{i}}$ : Valor del agua como insumo en el sector i (US $\left.\$ / \mathrm{m}^{3}\right)$

$Q_{i}^{d}$ : Volumen de agua demandado en el sector i (m³/año)

\section{Resultados de la valoración económica-ecológica}

\section{Valor de captación hídrica de ecosistemas}

Para aplicar el enfoque del "costo de oportunidad", se toma como referencia el nivel de rentabilidad de la agricultura, sobre todo, aquella que está basada en granos básicos (maíz, frijol, arroz y sorgo), que es la ocupación del suelo que compite con los ecosistemas forestados en las zonas de recarga de la subcuenca, como puede verse en la figura 2. A partir de la información del MAG (2013a y 2013b) referida a los costos de producción e índices de precios al productor agropecuario, se determina la rentabilidad promedio de Ios cuatro cultivos, la cual asciende a US\$106.0 ha/año, ponderando la superficie sembrada de cada uno de ellos. Este monto se incorpora en la ecuación 1 para calcular el valor de captación hídrica de los bosques y el cafetal, una vez se adicionen los costos de administración y mantenimiento de las actividades forestales y agroforestales, por lo que el valor total ascendería a US\$114.86.

\begin{tabular}{|c|c|}
\hline $\begin{array}{l}\text { Figura } 2 \\
\text { Localización de las zonas de recarga hídrica }\end{array}$ & $\begin{array}{c}\text { Figura } 3 \\
\text { Uso del suelo en las zonas de recarga hídrica }\end{array}$ \\
\hline (1) & 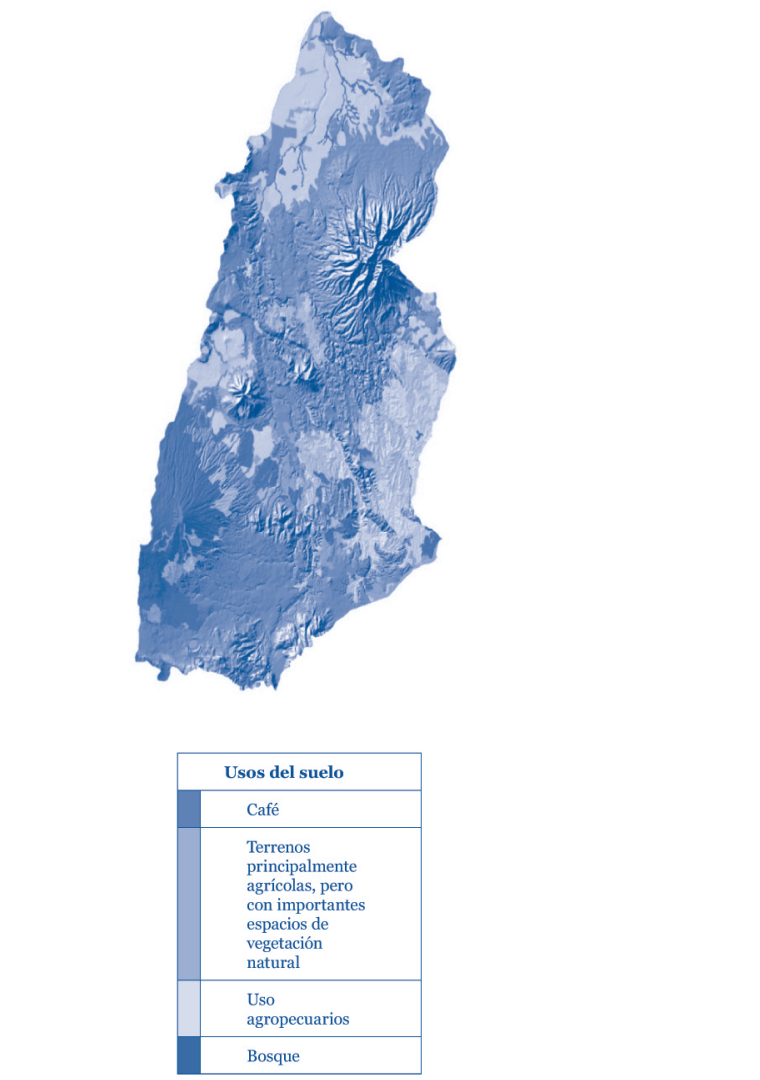 \\
\hline $\begin{array}{c}\text { Universidad Ténológica } \\
\text { de EI Salvador }\end{array}$ & Fuente: Elaboración propia \\
\hline
\end{tabular}


En condiciones socioeconómicas similares a la de la subcuenca del río Acelhuate, la importancia que la sociedad asigna al servicio ambiental hídrico respecto a los otros servicios ecosistémicos oscila entre $25 \%$ y $50 \%$, estableciéndose como un valor promedio $35 \%$; de manera que será esa la proporción del costo de oportunidad que debiera pagarse a los dueños de las áreas arboladas, a fin de que las mantengan ocupando los suelos. Por lo tanto, el valor monetario que define la compensación que se debe pagar por los usuarios del agua es de US\$77.30/ha/año. Entre tanto, el volumen de agua captado por la cobertura arbórea en la subcuenca es de 27.227.340 m³/año. Así, utilizando la ecuación 1, se obtiene el valor de captación (VC), el cual es de US $\$ 0.008 / \mathrm{m}^{3}$ :

$$
V C=\frac{0,35 \times 77,3 \times 7.938}{27.227 .340}=\mathbf{U S} \$ \mathbf{0 . 0 0 8} / \mathbf{m}^{\mathbf{3}}
$$

\section{Valor de restauración de la subcuenca}

La figura 3 muestra la superficie que se debe restaurar en la subcuenca, la cual corresponde a las tierras ubicadas en zonas de alta recarga hídrica (mayor a los $350 \mathrm{~mm}$ anuales) en donde el uso del suelo es agropecuario; este uso del suelo se compone de caña de azúcar, cultivos anuales, granos básicos, mosaico de cultivos y pastos, pastos naturales y pastos cultivados; esa superficie asciende a 12.704 hectáreas.

Según la Organización de las Naciones para la Alimentación y la Agricultura (FAO, 2010), las acciones efectivas de manejo sostenible de la tierra incluyen obras y prácticas de conservación de suelos y agua, agroforestería, diversificación de cultivos y manejo de bosques naturales, reforestación, capacitación y organización, desarrollo de actividades productivas sostenibles, manejo sostenible de recursos naturales, manejo de cuencas y desarrollo productivo agropecuario y empresarial. Con base en la FAO, la inversión para implantar este tipo de actuaciones es de US\$650 por hectárea por año.

A este valor habrá que sumarle el monto estimado de la utilidad generada por las actividades agrícolas de granos básicos, el cual asciende a US\$106.0/ha/año, dando como resultado un costo de US\$756.0; del cual se reconoce socialmente sólo el $35 \%$ como servicio ambiental hídrico. El resultado se multiplica por el área total a restaurar en la subcuenca, y se divide entre la productividad hídrica, que asciende a 27.227.340 m³/año. Con esta información se sustituyen las variables en la ecuación 2 para obtener el valor de restauración de US\$0,12/m³. Es decir,

$$
\begin{gathered}
V R=\frac{0,35 \times 756 \times 12.704}{27.227 .340} \\
V R=\mathbf{U S} \$ \mathbf{0 . 1 2} / \mathbf{m}^{3}
\end{gathered}
$$

\section{Valor del agua como insumo de la producción}

\section{$\checkmark$ Valor del agua en el sector doméstico}

Aunque en el sector doméstico no se produce un bien o servicio para el mercado, sí existe un precio por el bienestar que genera un consumo por encima del óptimo, en el que realmente no se satisfacen necesidades básicas (llenado de piscinas, lavado de automóviles, entre otros usos), por lo que debe estimarse un valor del agua para este sector.

En este sentido, la información de la Administración Nacional de Acueductos y Alcantarillados (Anda, 2011) es clave, sobre todo la que se refiere al nivel de consumo del sector residencial, distinguiendo entre: bajo, medio y alto. Asimismo, es importante conocer la tarifa de agua actual (según consumo), asumiendo un nivel de elasticidad precio de la demanda de -0.2518 , y un crecimiento económico de 2,1\% para el sector doméstico en los municipios de la subcuenca. Utilizando la ecuación 5 se obtiene el excedente del consumidor para los rangos de consumo en cuestión, obteniéndose los valores de la tabla 3. De estos, se consideran únicamente los consumos medios y altos, y se asocian con el nivel de demanda respectivo, dando como resultado que el valor promedio ponderado del agua en el sector doméstico equivale a US\$0.0073 por $\mathrm{m}^{3}$. 
Tabla 3

Valor del agua en el sector doméstico de la subcuenca del río Acelhuate

\begin{tabular}{|c|c|c|c|c|c|c|c|c|c|}
\hline \multirow{2}{*}{$\begin{array}{l}\text { Nivel de } \\
\text { consumo }\end{array}$} & \multicolumn{2}{|c|}{ Consumo } & \multicolumn{2}{|c|}{ Precio } & \multirow{2}{*}{ Elasticidad } & \multirow{2}{*}{$\begin{array}{c}\text { Parámetro } \\
\text { k }\end{array}$} & \multirow{2}{*}{$\begin{array}{c}\text { Tasa de } \\
\text { crecimiento }\end{array}$} & \multirow{2}{*}{$\begin{array}{c}\text { Parámetro } \\
\mathrm{k}_{1}\end{array}$} & \multirow{2}{*}{$\begin{array}{c}\text { Excedente } \\
\text { US } \$ / m^{3}\end{array}$} \\
\hline & $\mathbf{Q}_{1}$ & $Q_{2}$ & $P_{1}$ & $P_{2}$ & & & & & \\
\hline Baja & 11 & 11,17 & 0,210 & 0,210 & $-0,2518$ & 7,43 & 0,021 & 7,58 & 0.0004 \\
\hline Media & 30 & 30,47 & 0,319 & 0,326 & $-0,2518$ & 22,50 & 0,021 & 22,97 & 0.0016 \\
\hline Alta & 87 & 88,36 & 1,300 & 1,327 & $-0,2518$ & 92,94 & 0,021 & 94,89 & 0.0188 \\
\hline
\end{tabular}

Fuente: elaboración propia con base en registros de consumo y tarifas de los sistemas de acueducto residencial en la región metropolitana de San Salvador (Anda, 2011).

\section{$\checkmark$ Valor del agua en el sector industrial y comercial}

A diferencia del sector doméstico, en el industrial y comercial el agua sí constituye un insumo de producción de bienes y servicios con un precio de mercado (Calles, 2003), por lo que resulta de gran importancia el cálculo del valor monetario del agua en estos sectores, lo cual es posible si se trae a cuenta y se relacionan la demanda anual de agua de estos sectores y las tarifas hídricas que se les cobra, suponiendo una elasticidad-precio negativa y menor que la unidad.

De esta forma, para el cálculo de este valor se asume una elasticidad de $-0,3 \%$ y una tasa de crecimiento de 1,8 y 1,9
$\%$, para el sector industrial y comercial, respectivamente. Los datos que se muestran en la tabla 4 corresponden a la región metropolitana de San Salvador, y los cálculos utilizan la ecuación 5 , con niveles de consumo de agua ( $\mathrm{m}^{3} / \mathrm{mes}^{\mathrm{m}}$ y tarifas de agua cobradas por Anda (2011).

Con estos cálculos se obtienen los valores particulares de cada sector; no obstante, para ponderarlos resulta conveniente asociar dichos valores con los niveles de demanda anual; siendo así, el valor promedio del agua

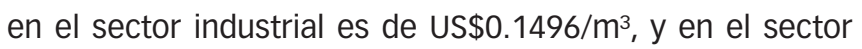

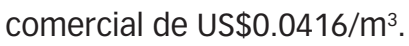

Tabla 4

Valor del agua en los sectores industriales y comerciales de la subcuenca del río Acelhuate

\begin{tabular}{|c|c|c|c|c|c|c|c|c|c|}
\hline \multirow{2}{*}{$\begin{array}{c}\text { Nivel de } \\
\text { demanda } \\
\left(\mathrm{m}^{3}\right)\end{array}$} & \multicolumn{2}{|c|}{ Consumo } & \multicolumn{2}{|c|}{ Precio } & \multirow{2}{*}{ Elasticidad } & \multirow{2}{*}{$\begin{array}{c}\text { Parámetro } \\
\text { k }\end{array}$} & \multirow{2}{*}{$\begin{array}{c}\text { Tasa de } \\
\text { crecimiento }\end{array}$} & \multirow{2}{*}{$\begin{array}{c}\text { Parámetro } \\
\mathrm{k}_{1}\end{array}$} & \multirow{2}{*}{$\begin{array}{c}\text { Excedente } \\
\left(\mathrm{US} \$ / \mathrm{m}^{3}\right)\end{array}$} \\
\hline & $\mathbf{Q}_{1}$ & $Q_{2}$ & $P_{1}$ & $P_{2}$ & & & & & \\
\hline \multicolumn{10}{|c|}{ Industrial } \\
\hline 0 a 20 & 7 & 7,09 & 2,085 & 2,12 & $-0,2518$ & 8,42 & 0,018 & 8,57 & 0,0018 \\
\hline 21 a 40 & 25 & 25,34 & 0,847 & 0,862 & $-0,2518$ & 23,98 & 0,018 & 24,41 & 0,0026 \\
\hline 41 a más & 866 & 877,64 & 1,452 & 1,478 & $-0,2518$ & 951,26 & 0,018 & 968,39 & 0,1527 \\
\hline \multicolumn{10}{|c|}{ Comercial } \\
\hline 0 a 20 & 9 & 9,13 & 2,085 & 2,12 & $-0,2518$ & 10,83 & 0,019 & 11,03 & 0,0025 \\
\hline 21 a 40 & 29 & 29,41 & 0,847 & 0,863 & $-0,2518$ & 27,81 & 0,019 & 28,34 & 0,0033 \\
\hline 41 a más & 254 & 257,60 & 1,452 & 1,480 & $-0,2518$ & 279,01 & 0,019 & 284,31 & 0,0500 \\
\hline
\end{tabular}




\section{$\checkmark$ Valor del agua en el sector agrícola}

En la subcuenca del río Acelhuate, el cultivo de caña de azúcar (localizado principalmente en los municipios de Nejapa y Apopa) satisface su demanda hídrica con una significativa proporción de agua bajo riego; de manera que la técnica del "cambio de productividad", para estimar el valor del agua en este sector, puede ser aplicada a partir de la información sobre el rendimiento anual por hectárea, producción, costos y precios de la caña de azúcar. Por lo tanto, aplicando la ecuación 6, se obtiene el valor del agua como insumo de la producción en el sector agrícola, el cual equivale US\$0.0099/ $\mathrm{m}^{3}$ (tabla 5).

Tabla 5

Valor del agua en el sector agrícola de la subcuenca del río Acelhuate

\begin{tabular}{|c|c|c|c|c|c|c|c|}
\hline \multirow{2}{*}{ Cultivo } & \multicolumn{2}{|c|}{$\begin{array}{l}\text { Rendimiento anual } \\
\text { (Tm/ha/año) }\end{array}$} & \multirow{2}{*}{$\begin{array}{c}\text { Agua usada } \\
\left(\mathrm{m}^{3} / \text { ha/año) }\right. \\
V_{r}\end{array}$} & \multirow{2}{*}{$\begin{array}{l}\text { Producción } \\
\mathrm{Kg} / \mathrm{m}^{3} \\
(Q r-Q s) / V r\end{array}$} & \multirow{2}{*}{$\begin{array}{l}\text { Costo de } \\
\text { producción } \\
\text { (US\$/Kg) } \\
\text { C }\end{array}$} & \multirow{2}{*}{$\begin{array}{c}\text { Precio } \\
\text { producto } \\
\text { (US\$/Kg) } \\
P\end{array}$} & \multirow{2}{*}{$\begin{array}{l}\left.\text { Valor del agua (US\$ } / \mathrm{m}^{3}\right) \\
(P-C) \times\left(Q_{r}-Q_{g}\right) / V_{1}\end{array}$} \\
\hline & $\begin{array}{c}\text { Secano } \\
\left(Q_{s}\right)\end{array}$ & $\begin{array}{l}\text { Riego } \\
{ }^{*}\left(Q_{r}\right)\end{array}$ & & & & & \\
\hline $\begin{array}{l}\text { Caña } \\
\text { de azúcar }\end{array}$ & 83.32 & 98.31 & $15.610,58$ & 0.96025 & 0.0246 & 0.035 & 0.0099 \\
\hline
\end{tabular}

Fuente: Elaboración propia con base en información de Goes/PNUMA (1981), MAG (2013a), MAG (2013b), Ramos (2010) y Agroindustria azucarera de El Salvador (2012).

Finalmente, se presentan en la tabla 6 los valores económicos de los componentes del servicio ambiental hídrico que proveen los bosques y cafetales de la subcuenca del río Acelhuate. Nótese que el valor de captación hídrica y de restauración es el mismo, independientemente del sector económico, ya que se espera que los usuarios en su conjunto reconozcan el servicio ecosistémico y establezcan un sistema de pago a los dueños de las tierras que se encuentran ocupadas por bosques y cafetales. En tanto que el agua como insumo de la producción en el sector industrial es el más alto (aproximadamente US\$0.15 por $\left.m^{3}\right)$, lo cual está en función del consumo del recurso que realizan las empresas dentro de este sector, así como por la tasa de crecimiento y los valores que se asuman para la elasticidad precio de la demanda. Este componente de la valoración es el que incide directamente para que sea el sector industrial el que deba reconocer un mayor valor del agua como servicio ambiental de los ecosistemas, el cual equivale a US\$0.28; seguido del sector comercial, con un valor de US\$0.17; y de los sectores agropecuario (US\$0.14) y doméstico (US\$0.13). 
Tabla 6

Valores económicos del servicio ambiental hídrico

\begin{tabular}{|l|c|c|c|c|}
\hline \multicolumn{1}{|c|}{ Sector } & $\begin{array}{c}\text { Valor de captación } \\
\text { US\$ } / \mathrm{m}^{3}\end{array}$ & $\begin{array}{c}\text { Valor de restauración } \\
\text { US } \$ / \mathrm{m}^{3}\end{array}$ & $\begin{array}{c}\text { Valor del agua como } \\
\text { insumo } \\
\text { US } \$ / \mathrm{m}^{3}\end{array}$ & $\begin{array}{c}\text { Total } \\
\text { US\$ } / \mathrm{m}^{3}\end{array}$ \\
\hline Doméstico & 0.008 & 0.12 & 0.0073 & $\mathbf{0 . 1 3 5 3}$ \\
\hline Industrial & 0.008 & 0.12 & 0.1496 & $\mathbf{0 . 2 7 7 6}$ \\
\hline Comercial & 0.008 & 0.12 & 0.0416 & $\mathbf{0 . 1 6 9 6}$ \\
\hline Agropecuario & 0.008 & 0.12 & 0.0099 & $\mathbf{0 . 1 3 7 9}$ \\
\hline
\end{tabular}

Fuente: elaboración propia.

\section{Conclusiones y recomendaciones}

Los ecosistemas que posibilitan la provisión de agua para los distintos sectores de la economía localizada en la subcuenca del río Acelhuate son el café y el bosque que se localizan principalmente en el volcán de San Salvador, Antiguo Cuscatlán y Santa Tecla, con importantes relictos en otros municipios de la subcuenca, que en conjunto ocupa no más del $10 \%$ del territorio, lo cual podría traducirse en una seria amenaza para la provisión del servicio ecosistémico; sobre todo, debido a que en la ocupación del suelo predominan las actividades agrícolas y urbanas que compiten por espacio con estos ecosistemas.

Por esta razón es fundamental que se implante una gestión territorial efectiva de las áreas de recarga, lo cual implica emprender esfuerzos de protección y restauración de ecosistemas, y que se involucre a todos los actores vinculados con el sector hídrico en la región metropolitana de San Salvador, entre los que destacan dueños de tierras, usuarios del agua (incluida Anda) e instituciones públicas relacionadas con la gestión hídrica.

Los valores monetarios, estimados a partir de la utilización de los distintos enfoques de valoración económica, demuestran que el servicio ambiental hídrico no tiene ningún reconocimiento por los usuarios del agua, ya que no son cobrados en las tarifas hídricas que se paga en cada sector de la economía, por lo que es claro que existe un subsidio ambiental en las actividades productivas. Estos valores monetarios deben de relacionarse directamente con la demanda de cada sector económico y focalizarse, sobre todo, en la población que utiliza el agua para satisfacer necesidades que no son básicas para el ser humano, cuyos volúmenes superan los $30 \mathrm{~m}^{3}$ por mes en el sector doméstico. Siendo así, el sector doméstico (o residencial) debiera pagar US $\$ 0.13$ por cada $\mathrm{m}^{3}$ de agua consumido; mientras que el sector industrial lo debería hacer con US $\$ 0.28 / \mathrm{m}^{3}$; el comercial con US $\$ 0.17 / \mathrm{m}^{3}$; y el sector agropecuario con US $\$ 0.14 / \mathrm{m}^{3}$.

Las distintas técnicas desarrolladas en este artículo podrán ser consideradas en el contexto de una posible aprobación de la ley general de aguas, sobre todo en lo que respecta al cálculo de cánones por aprovechamiento de agua, como un mecanismo para reconocer el valor del agua como servicio que proveen los ecosistemas boscosos y cafetales de la subcuenca. No obstante, en un escenario sin aprobación de una ley de aguas, deberá fortalecerse a los gobiernos locales para que sean estos, amparados en la Constitución de la República y el Código Municipal, los que, mediante el diseño y aplicación de ordenanzas, gestionen el recurso hídrico, haciendo uso de esquemas de cobro y pago por servicios ecosistémicos; o bien, empleando incentivos para aquellos usuarios que estén alineados con los objetivos de restauración y conservación de ecosistemas que favorezcan la captación y disposición del recurso hídrico a la sociedad.

\section{Referencias}

Administración Nacional de Acueductos y Alcantarillados [Anda] (2011). Boletín estadístico. República de El Salvador, C.A.

Agroindustria Azucarera de El Salvador. 2012. Foro: "Agricultura sostenible, estrategia de negocios para 
nuevos mercados". Fundación Empresarial para la Acción Social, Fundemas. El Salvador.

Barrantes, G. y Vega, M. (2002). “Evaluación del servicio ambiental hídrico en la cuenca del río Tempisque y su aplicación al ajuste de tarifas". Asociación para el Desarrollo Sostenible del Área de Conservación Tempisque (Asotempisque). Costa Rica.

Calles, J. (2003). “Evaluación del servicio ambiental hídrico en la Cuenca Alta del Río Lempa y su aplicación en el ajuste de la tarifa hídrica del Área Metropolitana de San Salvador, El Salvador". Tesis Mag. Sc. Turrialba, CR, Catie. $123 \mathrm{p}$.

Catie (1997). "Productos no maderables del bosque en Baja Talamanca, Costa Rica". Olafo-Cifor. Asociación San Migueleña de Conservación y Desarrollo (Asacode). Turrialba, Costa Rica.

CCT-Cinterpeds (1995). "Valoración Económico Ecológica del Agua: Primera Aproximación para la Interiorización de Costos". San José, Costa Rica.

Fundación Salvadoreña para la Investigación del Café [Procafe] (2009). "El café, generador de servicios ambientales". Fundación Procafe. Santa Tecla, La Libertad. El Salvador.

Goes [Gobierno de El Salvador])/PNUMA [Programa de las Naciones Unidas para el Medio Ambiente] (1981). "Plan Maestro de Desarrollo y Aprovechamiento de Ios Recursos Hídricos. El Salvador, Documento Básico No. 12, Recursos y Demandas Potenciales de la Región
'A' Cuenca Alta". Plamdarh, San Salvador, El Salvador. $196 \mathrm{p}$.

Heuveldop J. et al. (1986). Agroclimatología tropical. 1era Ed. Editorial Uned. San José, Costa Rica.

Jobbágy, E. (2009). Servicios hídricos de los ecosistemas y su relación con el uso de la tierra en la llanura chacopampeana. Ediciones Inta, Buenos Aires.

Martínez, M. (2008). "Mercados de servicios ambientales. Análisis de tres experiencias centroamericanas de pago por servicios ambientales". Tesis doctoral para el programa de ciencias ambientales (economía ecológica y gestión ambiental). Universidad Autónoma de Barcelona.

Ministerio de Agricultura y Ganadería [MAG] (2013a). Costos de producción 2012-2013. Dirección General de Economía Agropecuaria. División de Estadísticas Agropecuarias. República de El Salvador, C.A.

(2013b). Anuario de estadísticas agropecuarias 2012-2013. Dirección General de Economía Agropecuaria. División de Estadísticas Agropecuarias. República de El Salvador, C.A.

Organización de las Naciones Unidas para la Alimentación y la Agricultura [FAO] (2010). "Evaluación de los recursos forestales mundiales. Informe nacional El Salvador". FAO, Departamento Forestal. Roma 00153, Italia.

Ramos, C. (2010). "Análisis de los efectos de la producción de cultivos bioenergéticos sobre la disponibilidad de los recursos hídricos: el caso del sistema chira". Universidad Nacional Agraria La Molina. Perú. 